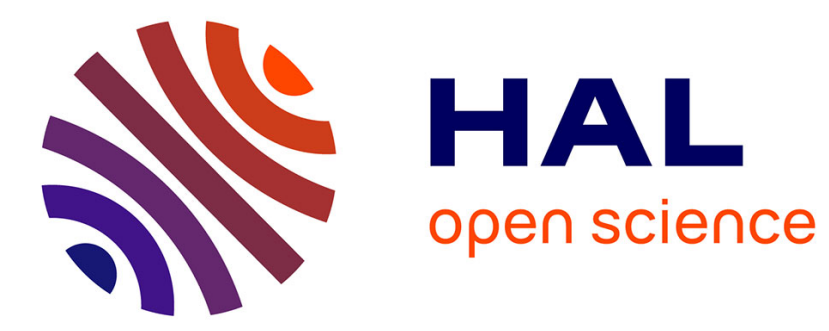

\title{
La formation des agents de la Fonction publique au miroir du secteur privé \\ Coralie Perez
}

\section{To cite this version:}

Coralie Perez. La formation des agents de la Fonction publique au miroir du secteur privé. Formation Emploi. Revue française de sciences sociales, 2003, janvier-mars (81), pp.82-97. halshs-00375035

\section{HAL Id: halshs-00375035 https://shs.hal.science/halshs-00375035}

Submitted on 15 Apr 2009

HAL is a multi-disciplinary open access archive for the deposit and dissemination of scientific research documents, whether they are published or not. The documents may come from teaching and research institutions in France or abroad, or from public or private research centers.
L'archive ouverte pluridisciplinaire HAL, est destinée au dépôt et à la diffusion de documents scientifiques de niveau recherche, publiés ou non, émanant des établissements d'enseignement et de recherche français ou étrangers, des laboratoires publics ou privés. 


\title{
LA FORMATION DES AGENTS DE LA FONCTION PUBLIQUE AU MIROIR DU SECTEUR PRIVÉ
}

\author{
Par Coralie Perez
}

Encore assez peu étudiée par les économistes, la fonction publique représente un quart de l'emploi salarié en France et est actuellement soumise à des tensions internes (départs en retraite massifs, évolution des missions de service public et du statut...) et externes (concurrence avec le secteur privé pour de futurs recrutements de diplômés, problème de fidélisation des agents). Dans ce contexte, la formation continue pourrait jouer un rôle croissant. Mais on sait peu de choses sur les pratiques de formation dans ce secteur, notamment par rapport au secteur privé. La vision souvent monolithique de l'emploi public, réduit à l'emploi statutaire, conduit tant à minorer son hétérogénéité (en termes de qualification, de mode de gestion de la main-d'œuvre, de statut) qu'à sous-estimer les proximités et les échanges entre les deux secteurs d'emploi. Ainsi, les dispositions législatives et réglementaires encadrant le droit à la formation des fonctionnaires ont été élaborées en référence au secteur privé. Dès lors, les agents de la fonction publique disposent formellement des mêmes dispositifs. Dans ce cadre, l'enquête «Formation continue 2000 » ( $c f$. introduction au dossier) nous permet d'en éclairer le recours et les usages dans chacun des deux secteurs d'emplois. En opposant (et rapprochant) le recours et les pratiques de formation du public et du privé, on contribue certes à durcir le clivage entre «gens du privé » et «gens du public » (Singly, Thélot, 1988). Mais l'hypothèse sous-jacente est que chacun d'eux pourrait définir un espace de libertés et de contraintes propres à engendrer des comportements spécifiques à l'égard de la formation. Lorsqu'on cherche à expliquer un accès plus large des agents de la fonction publique à la formation, deux résultats émergent. D'une part, la participation à la formation est globalement moins inégalitaire dans la fonction publique et dépend partiellement de la spécificité des fonctions et des métiers exercés. D'autre part, si la fonction publique et le secteur privé se distinguent, c'est moins par les modalités de formation que par l'existence d'un environnement plus ou moins propice à la connaissance de ses droits en matière de formation et à leur exercice.

\section{LA FORMATION CONTINUE, ANALYSEUR DES SPÉCIFICITÉS DES MARCHÉS DU TRAVAIL PUBLICS}

Analyser et comparer le rôle de la formation continue dans la fonction publique par rapport au secteur privé peut sembler peu pertinent. En effet, la fonction publique est souvent perçue comme une entité homogène, possédant une logique de fonctionnement propre, apte à soustraire ses fonctionnaires aux tensions économiques. Dans ce cadre, la formation continue ne jouerait qu'un rôle mineur dans le déroulement d'une carrière régie par l'ancienneté, voire constituerait une échappatoire au travail sans fondement utile (Rouban, 2001a). Or, cette image est trompeuse pour plusieurs raisons. Tout d'abord, le droit à la formation des fonctionnaires est bien établi et a été calqué sur celui des salariés du secteur privé (1.1.). Ensuite, si la formation continue dans le public présente bien des caractéristiques liées aux

\footnotetext{
* Coralie Perez est économiste, chargée d'études au Département Formation Continue du Céreq. Ses travaux portent sur l'évaluation des politiques d'emploi et de formation, ainsi que sur la formation continue en lien avec le fonctionnement des marchés du travail. Elle a notamment publié en 2001, Évaluer les programmes d'emploi et de formation, l'expérience américaine, La Documentation Française Dossier du Centre d'études de l'emploi $\mathrm{n}^{\circ} 18$, et plus récemment, avec Marion Lambert et Philippe Zamora, 2002, « La formation continue : un accès très inégal », Données Sociales, Insee.
} 
principes d'organisation des carrières, elle demeure comparable à celle mise en œuvre dans le secteur privé (1.2.). Enfin, à l'instar du secteur privé, la fonction publique présente une diversité de qualifications, de métiers et de statuts associés à des besoins de formation divers et inégaux. Dans un contexte de changements technologiques, de recomposition des missions, de diversification des systèmes professionnels, la fonction publique est soumise à des tensions comparables à celles qui s'exercent dans le secteur privé. Face à elles, la formation continue pourrait être amenée à jouer un rôle stratégique (1.3.).

\subsection{Un droit à la formation calqué sur le secteur privé}

Le droit à la formation continue des fonctionnaires ne se réduit pas à la préparation aux concours. Formellement, agents de la fonction publique et salariés du privé sont dotés des mêmes outils. La loi du 16 juillet 1971 pose les principes généraux du droit à la formation des fonctionnaires en même temps qu'elle définit le droit à la formation des salariés du commerce et de l'industrie. Elle stipule notamment que «l'État met en auvre au bénéfice de ses agents une politique coordonnée de formation professionnelle et de promotion sociale semblable par sa portée et les moyens employés à celle destinée aux salariés du secteur privé $\rangle^{1}$.

Toutefois, il faudra attendre la circulaire du 23 février 1989, et l'accord cadre qui en découle, pour que s'affirme véritablement le droit à la formation des fonctionnaires; elle fait de la formation un «instrument privilégié » au service d'une "nouvelle politique des relations de travail 》 dans la fonction publique (Lenoir, 1996). Au sein d'un projet politique de modernisation de l'État, cette circulaire inscrit la formation comme un outil de la «gestion prévisionnelle des emplois et des compétences » visant à adapter les agents à l'évolution de leurs missions. Consécutivement, plusieurs accords-cadres triennaux, destinés à être appliqués et déclinés localement, sont signés dans les années $1990^{2}$. Leur examen fait apparaître une tentative (voire « une tentation ? » s'interroge H. Lenoir) de transposition au secteur public des dispositions prises dans le secteur privé.

\section{$<$ ENCADRE 1 ici>}

Ainsi, dans un souci de mesure et de comparaison avec le privé, une exigence de quantification de l'effort de formation est formulée. L'accord-cadre du 10 juillet 1992 fixe d'ailleurs l'effort de formation à atteindre dans la fonction publique d'État en référence à la moyenne de celui consenti à la formation continue à cette époque par les entreprises privées assujetties à l'obligation légale ( $3,2 \%$ de la masse salariale en 1992). Les modalités de réalisation de cet engagement sont spécifiques à chaque fonction publique. Alors que le respect des objectifs définis par l'accord-cadre relève de chaque ministère dans la Fonction publique d'État, la Fonction publique hospitalière s'est dotée d'un organisme paritaire collecteur agréé l'ANFH (l'Association nationale pour la formation permanente du personnel hospitalier), le CNFPT (Centre national de la fonction publique territoriale) assurant ce rôle dans la Fonction publique territoriale.

Comme dans le secteur privé, le plan de formation, réalisé à l'initiative de l'employeur, est au cour du dispositif de formation des fonctions publiques et les agents sont tenus, comme leurs homologues du privé, de se former pour assurer leur adaptation à l'emploi ${ }^{3}$. Progressivement, les agents de la fonction

\footnotetext{
${ }^{1}$ Art.41 à 43, JO du 17-7-71.

${ }^{2}$ Le dernier accord-cadre a été signé en 1996 et aucune nouvelle négociation n'a repris depuis son échéance. Une évaluation de la politique de formation de l'État, préalable a une reprise des négociations, a été confiée en mars 2001 à une instance d'évaluation présidée par J.-F. Cuby. Cette instance, créée par le Conseil national de l'évaluation, doit rendre ses conclusions au début de l'année 2003.

${ }^{3}$ H. Lenoir signale cependant que les modalités de recours sont plus favorables aux agents de la fonction publique puisque confrontés à trois refus, ces derniers peuvent saisir une commission administrative paritaire
} 
publique sont dotés des mêmes dispositifs, tels que le congé de formation professionnelle (qui s'apparente au congé individuel de formation du secteur privé) ou encore le bilan professionnel, équivalent fonctionnel du bilan de compétences.

La similitude dans les modalités juridiques organisant la formation continue dans les deux secteurs reflète en partie celle des finalités des actions de formation: l'adaptation au premier ou au nouvel emploi, le maintien ou le perfectionnement de la qualification professionnelle, l'acquisition de qualifications plus élevées et l'affirmation du principe d'une gestion plus individualisée au travers du bilan de compétences (ou bilan professionnel). Peu d'innovations propres à la fonction publique sont donc intervenues; " la fonction publique qui est appame longtemps comme la locomotive des avancées sociales, en innervant par contagion le droit privé, apparaît $[\ldots]$ à la remorque du livre $I X d u$ Code du travail [en matière de formation professionnelle] » (Lenoir, 1996).

La construction progressive du dispositif de formation des agents de la fonction publique sur le modèle du secteur privé est un facteur encourageant la comparaison dans ce domaine. Il faut à présent identifier les caractéristiques propres à la formation continue dans la sphère publique influencées par certaines propriétés des marchés du travail publics.

\subsection{Les spécificités de la formation dans la fonction publique}

Dans la fonction publique, la formation initiale (formation professionnelle initiale) comprend les actions qui donnent à l'agent accédant à un emploi une formation professionnelle à la fois théorique et pratique visant à l'y préparer. Ces actions de formation concernent des agents venant de réussir un concours interne ou externe, des fonctionnaires promus dans un nouveau corps ou bien encore des contractuels en période d'essai. Dans certains cas, l'entrée dans la fonction publique est subordonnée à l'accomplissement d'une telle formation. La formation continue au sens de la fonction publique comprend, quant à elle, les actions de formation tout au long de la carrière de l'agent, titulaire ou non : les actions de perfectionnement, d'adaptation et de maintien des qualifications, les actions de préparation aux concours ( $c f$. infra), les congés de formation ainsi que le bilan professionnel. Cette distinction formelle se traduit aussi sur le plan organisationnel puisque les formations professionnelles initiales sont généralement dispensées au sein de structures ad hoc (écoles de formation administratives par exemple...) tandis que la formation continue peut être délivrée, outre dans ces écoles, au sein des ministères (structures propres) ou déléguée à d'autres prestataires (centres de gestion...).

Ainsi, bien que recrutant des personnes en moyenne plus diplômées que le secteur privé par des modes de sélection qui, pour une part, redoublent la sélection opérée par la voie scolaire et n'accordent que peu de place à l'expérience professionnelle, la fonction publique organise la formation des nouvelles recrues pour des durées plus ou moins longues. Cette pratique, quasi unique en Europe, (à l'exception de l'Espagne), actuellement discutée au sein de l'administration, pourrait expliquer le peu d'intérêt traditionnellement accordé à la formation continue dans la fonction publique française (Rouban, 1995).

La singularité de la formation professionnelle initiale ne traduit pas nécessairement un particularisme des pratiques ; c'est une convention d'enregistrement des formations qui n'entrave pas la comparaison avec le secteur privé. . Quant aux pratiques enregistrées sous ce vocable, elles font écho au constat effectué par P. Béret selon lequel, compte tenu des ressources dont disposent les jeunes actifs, «la

pour opposer un recours. Cette possibilité n'existe pas dans le privé où l'employeur «n'est jamais obligé de répondre à une demande individuelle (dans le cadre du Plan) et peut s'opposer au départ en formation [...] sans être tenu de motiver son refus » (1996).

${ }^{4}$ L'enquête «Formation Continue 2000 » s'adressait indifféremment aux salariés du privé et du public. La consigne donnée aux enquêteurs lors du recueil de l'information était de recenser toutes les formations, financées ou non par l'employeur, relevant ou non du plan de formation. Le nombre de formations n'est donc pas a priori sous estimé dans la fonction publique. 
formation continue ne fait que ratifier en l'objectivant, le succès relatif du salarié dans l'épreuve de sélection » (2002). Ce faisant, elle succèderait à une promotion plus souvent qu'elle ne la précèderait.

Les préparations aux concours représentent quant à elles une forme spécifique de pratiques de formation continue dans la fonction publique. Cette singularité est liée au principe d'avancement de carrière. En effet, la réussite d'un concours constitue le seul moyen de contourner la règle de l'ancienneté pour obtenir une promotion de grade ou changer de corps (cf. encadré). Prenant la forme de stage ou de cours par correspondance, ces préparations peuvent se dérouler tout ou partie pendant les heures de services. Ainsi, l'expérience cumulée au cours de l'activité professionnelle et d'actions de formation continue ne suffit généralement pas à s'élever dans la hiérarchie administrative. Parallèlement, la progression salariale est en partie déconnectée de parcours de formation organisés collectivement et répond d'abord à l'ancienneté dans l'emploi. Pour ces raisons, on ne pourrait accorder à la formation continue qu'un rôle mineur, à l'exception de ces préparations aux concours. Si ces dernières constituent une part significative des actions de formation continue des agents de la fonction publique ${ }^{5}$, elles ne s'y réduisent pas.

En fait, tous les agents ne sont pas régis par le statut général des «fonctionnaires », principe organisateur des recrutements, des mobilités et des rémunérations commun aux trois fonctions publiques. De plus, la diversité des qualifications et des métiers exercés concourt aussi à structurer l'emploi public. La diversité de ses caractéristiques conduit même à poser l'hypothèse de la banalisation de l'emploi public au sens d'une convergence de ses caractéristiques avec celles de l'emploi privé (Commissariat Général du Plan, 1994, Rouban 1995). En outre, les marchés du travail publics ne sont pas étanches et les tensions qui s'exercent dans et hors la fonction publique pourraient accorder à la formation un rôle plus important dans la gestion des mobilités

\subsection{Marchés du travail public et privé : des tensions comparables ?}

Loin d'être homogène, l'emploi public présente des différences internes importantes qui semblent démentir l'existence de deux «mondes » distincts et de rapports différenciés à la formation. Ainsi, les structures d'emploi par qualification des trois fonctions publiques sont différentes. La Fonction publique d'État se distingue par le niveau élevé de formation de ses agents (les titulaires d'un diplôme de bac +3 et plus représentent près du tiers des agents) tandis que dans la Fonction publique hospitalière, plus du tiers des agents sont titulaires d'un bac +2 . Quant à la Fonction publique territoriale, la répartition par niveau de diplôme des agents est identique à celle du secteur privé ( $c f$. graphique 1). Cette répartition des agents par niveau de formation, qui se reflète dans celle des emplois par catégorie ${ }^{6}$, conduit à formuler plusieurs interrogations quant à l'équité du recours à la formation. L'accès à la formation est-i1, comme dans le secteur privé, fortement lié au niveau de qualification détenu par le salarié ? Dans ce cas, doit-on s'attendre à des inégalités d'accès de même intensité ? Proche du secteur privé par sa distribution des qualifications, la Fonction publique territoriale présente-t-elle des comportements de formation qui lui sont comparables?

\section{$<$ INS GRAPH 01>}

Les fonctions et les métiers exercés sont également divers entre les fonctions publiques. Ainsi, le personnel enseignant représente plus de la moitié des effectifs de la Fonction publique d'État tandis que

\footnotetext{
${ }^{5}$ Dans la Fonction publique d'État, elles représentent $17 \%$ des actions de formation continue et constituent le second poste après les actions de perfectionnement (en nombre de jours, hors congé de restructuration ; DGAFP, 2001).

${ }^{6}$ Près de $40 \%$ des agents de la Fonction publique d'État sont en catégorie A, $35 \%$ des agents de la Fonction publique hospitalière en catégorie $\mathrm{B}$ et près de $80 \%$ des agents de la Fonction publique territoriale relèvent de la catégorie $\mathrm{C}$ ( $c f$. encadré 1).
} 
la Fonction publique hospitalière est composée d'une centaine de métiers techniques et réglementés, dont l'évolution est rapide en raison des progrès techniques et médicaux. La Fonction publique territoriale, dont le taux d'encadrement est proche de celui du secteur privé, recouvre des métiers techniques de qualification moyenne mais susceptibles d'évoluer dans la perspective d'une décentralisation accrue. Cette diversité a des implications sur la participation à la formation : les contraintes pesant sur elle, de même que les nécessités d'adaptation à l'évolution de l'emploi étant liées à la technicité des domaines d'activité ainsi qu'à la taille des ministères et des établissements d'exercice.

Diversité aussi dans les modes de gestion du personnel puisque au-delà des principes communs qui les régissent (carrière structurée en corps ou cadres d'emplois, recrutement par concours), chaque fonction publique assure de façon spécifique la gestion de ses ressources humaines, et donc la formation continue. Au sein de la Fonction publique d'État, et à l'exception de trois corps, la gestion de la maind'œuvre est propre à chaque ministère. Dans la Fonction publique territoriale, la gestion des emplois est décentralisée, de même que dans la Fonction publique hospitalière où chaque établissement gère son personnel; ces modes de gestion contribuant à définir des espaces de mobilité (fonctionnelle, promotionnelle, géographique) différenciés (Ministère de la Fonction publique, 2001).

Enfin, la diversité de statut devrait être prise en compte puisque le statut de fonctionnaire n'est pas l'apanage de tous les agents de la Fonction publique. Coexistent ainsi des agents aux statuts plus ou moins précaires ${ }^{7}$. Les non-titulaires représentent près d'un quart des effectifs de la fonction publique, la part des contractuels variant de $14 \%$ dans la Fonction publique d'État à $23 \%$ dans la Fonction publique territoriale. L'embauche de ces salariés répond à des préoccupations de nature diverse. L'emploi de non-titulaires permet d'atténuer, voire de contourner, les contraintes posées par les règles de recrutement et de mobilité interne à la fonction publique, et de pallier l'absence de créations d'emplois statutaires en nombre suffisant. En corollaire, le recours ponctuel à des qualifications spécifiques ne relevant pas des métiers exercés traditionnellement par les titulaires, peut également motiver leur embauche (Desbarats, 2001). Enfin, le nombre d'emplois non titulaires renvoie au rôle joué par l'employeur public dans la politique publique d'emploi, notamment au travers des emplois aidés de type contrat emploi-solidarité ou «Emploi jeunes ». Prenant acte de cette diversité, on peut s'interroger sur la situation des «précaires » de la fonction publique au regard de la formation : sont-ils mieux lotis que leurs homologues du secteur privé ? En effet, on peut s'attendre à ce que la fonction publique - moins soumise à la pression de la concurrence - conduise une politique plus équitable envers ses agents eu égard à leur statut.

À cette diversité qui invite à évoquer un ensemble de marchés internes publics, s'ajoute la perméabilité des marchés du travail privés et publics. Si les marchés internes privés font l'objet de déstabilisation exprimée par les tentatives de déconnexion entre rémunération et ancienneté (Germe, 2001 ; Gautié, 2002), la fonction publique n'est pas à l'abri des tensions à l'œuvre dans l'économie. Tout d'abord, la fonction publique a pu apparaitre comme un refuge pour des jeunes diplômés à une période où le taux de chômage et l'insécurité de l'emploi étaient élevés. De nombreux diplômés occupent ainsi des emplois relativement peu qualifiés (Goux, Maurin, 1993). Ce phénomène de «déclassement» (ou de « surdiplôme ») risque d'aviver la concurrence interne pour les mobilités verticales et de pénaliser les agents les moins qualifiés ou les plus âgés (Kopel, 2001). Par ailleurs, on peut s'interroger sur la capacité de la fonction publique à attirer et retenir les futurs diplômés, la concurrence avec le secteur privé pouvant être aiguë pour certaines qualifications. Enfin, la proximité de départs en retraite massifs donne encore plus d'acuité aux problèmes de recrutement et de mobilité. Ces tensions pèsent sur les règles en vigueur et dans ce contexte, la formation continue pourrait être amenée à jouer un rôle « stratégique » pour fidéliser les nouvelles recrues, atténuer les difficultés de recrutement externe et favoriser l'organisation des mobilités internes, tant verticales qu'horizontales.

\footnotetext{
Il faut en effet distinguer la situation des contractuels les plus favorisés (collaborateurs d'élus locaux, directeurs de service recrutés sur contrat temporaire...) de celle qui cumule l'absence de stabilité et des emplois peu rémunérateurs (salariés embauchés sous contrats aidés...).
} 
La suite de ce travail vise à mieux cerner les pratiques de formation dans la fonction publique au miroir de celles, mieux connues, du secteur privé. La proximité des instruments légaux et les évolutions des marchés du travail publics autorisent en effet la mise en regard du recours et des usages de la formation continue dans deux sphères désignant a priori des espaces de régulation et de contraintes distincts. Outre une meilleure connaissance des pratiques dans la sphère publique, l'intérêt est de tester l'existence de comportements de formation propres aux agents de la fonction publique. Trois dimensions sont successivement explorées : le recours (fréquence et intensité), les modalités et contenus de formation, et le rapport à la formation (initiative, connaissance des droits).

\section{UNE PARTICIPATION À LA FORMATION PLUS FORTE ET MOINS INÉGALITAIRE ${ }^{8}$}

\subsection{Les agents publics se forment davantage et plus longtemps}

Selon les données de l'enquête « Formation Continue 2000 », $47 \%$ des agents de la fonction publique ont suivi au moins une formation entre janvier 1999 et février 2000, contre près d'un tiers des salariés du privé. ${ }^{9}$ La participation à la formation est particulièrement élevée dans la Fonction publique d'État et la Fonction publique hospitalière où la moitié des agents sont partis en formation sur la période ; la Fonction publique territoriale présente quant à elle un taux d'accès proche de celui du secteur privé.

\section{$<$ INS GRAPH 02>}

Plus fréquente, la formation continue des agents de la fonction publique est également plus intensive. Si $60 \%$ des formations durent moins de 24 heures (environ trois jours) dans les deux secteurs, les formations de une à deux semaines sont plus fréquentes dans la fonction publique que dans le secteur privé ( $c f$. graphique 2). De plus, sur la période étudiée de quatorze mois ${ }^{10}$, les salariés du public se distinguent par la possibilité qui leur est offerte de passer un temps plus long en formation que leurs homologues du secteur privé ( $c f$. tableau 1).

\section{$<$ INS TAB 01>}

Ce constat n'est pas surprenant compte tenu des objectifs initiaux de la formation professionnelle dans la fonction publique, énoncés dans l'accord-cadre du 22 février 1996: la promotion sociale et la préparation des concours internes. L'atteinte de ces objectifs nécessite des durées de formation conséquentes.

L'accord du 22 février 1996 affiche également une préoccupation particulière envers les agents les plus éloignés de la formation en stipulant des durées minimales de formation selon la catégorie d'appartenance ${ }^{11}$. Nos données nous permettent de comparer la distance à la formation des salariés des

\footnotetext{
${ }^{8}$ À partir de ce point (et hormis les modèles), une partie des résultats statistiques présentés sont issus d'un rapport réalisé pour l'instance d'évaluation de la politique de formation continue des agents de l'État (Lowezanin, Perez, 2002).

${ }^{9}$ Seules les entreprises de 500 salariés et plus présentent un taux d'accès à la formation équivalent à celui de la Fonction publique.

${ }^{10}$ Afin de tenir compte de la possible récurrence des épisodes de formation, on calcule la durée cumulée des formations sur la période d'observation (quatorze mois). Cette récurrence est faible pour les formés du public et du privé. Plus de la moitié d'entre eux n'ont suivi qu'une action de formation et près de $20 \%$ des formés déclarent deux formations entre janvier 1999 et février 2000.

${ }^{11}$ L'accord énonce cinq jours de formation pour les agents de catégorie A et B, et six jours pour ceux de catégorie $\mathrm{C}$, répartis sur les trois ans couverts par l'accord.
} 
deux secteurs. Quatre agents de la fonction publique sur dix déclarent ne pas avoir suivi de formation depuis deux ans. Les titulaires d'emploi de catégorie $\mathrm{C}$ paraissent les plus éloignés de la formation puisque la moitié d'entre eux sont dans cette situation. Comparés à la fonction publique, les salariés du privé semblent moins bien lotis. Ainsi, ils sont proportionnellement plus nombreux à n'avoir suivi aucune formation depuis deux ans $(60 \%)$. Dans les deux secteurs, les moins qualifiés (ouvriers et employés) déclarent plus fréquemment que les cadres être dans cette situation (34\% des cadres contre $63 \%$ des employés et $73 \%$ des ouvriers).

Relativement moins éloignés de la formation, les agents de la fonction publique sont amenés à suivre des formations plus longues que leurs homologues du privé. Ces formations sont a priori plus qualifiantes et devraient donc avoir un impact sur le déroulement de carrière de l'agent. Plusieurs travaux mettent en évidence, sur longue période, un accès plus large à la formation, parallèlement à la réduction des durées des formations (Bentabet, Gauthier, Marion, 2001 ; Goux, Zamora, 2001). Ce mouvement exprimerait le tassement des formations promotionnelles au profit des formations «professionnelles », davantage liées à la gestion de l'emploi dans une logique de court terme, ainsi que la raréfaction des chances de promotion (Podevin, 1999). La fonction publique résisterait mieux à cette tendance en continuant à offrir à ses agents la possibilité de suivre des formations longues ; ces dernières assurant encore ainsi, davantage que dans le privé, une fonction de régulation sur les marchés internes.

À ce stade, la fonction publique semble représenter un environnement plus propice au suivi d'une formation continue. Nous allons à présent tenter d'expliquer ce différentiel de participation à la formation. La fonction publique est-elle intrinsèquement plus «généreuse » que le secteur privé ou des facteurs «objectifs » permettent-ils de mieux comprendre ces résultats ? Une première hypothèse est que la participation à la formation reflète les structures d'emploi par qualification du public et du privé.

\subsection{Fonction publique : des agents plus qualifiés donc plus formés ?}

L'assertion selon laquelle « la formation va à la formation » est corroborée par de nombreuses études. En ce sens, la plus grande participation à la formation des agents de la fonction publique traduirait leur niveau plus élevé de formation initiale et la part relativement plus grande des cadres et professions intermédiaires sur les marchés du travail publics. Ceci expliquerait que la Fonction publique territoriale, qui présente une distribution des agents par niveau de diplôme et catégorie d'emploi similaire à celle du secteur privé, ait également un taux d'accès et des durées de formation plus proches des salariés du privé que des agents des autres fonctions publiques.

Pour éprouver cette hypothèse, nous avons utilisé un modèle prenant l'appartenance à un secteur d'emploi (fonction publique ou secteur privé) comme un facteur explicatif de la probabilité d'avoir suivi une formation, l'influence d'autres facteurs sur cette probabilité, tels que la catégorie socioprofessionnelle, l'âge, le sexe, le statut d'emploi..., étant contrôlée. Ainsi, «toutes choses égales par ailleurs », l'appartenance à la fonction publique exerce une influence positive et significative sur la probabilité de suivre une formation. Afin de déterminer si ce résultat masque des différences internes à la sphère publique, les trois fonctions publiques sont ensuite introduites pour tester, concurremment avec le secteur privé, leur influence sur la probabilité de suivre une formation. "Toutes choses égales par ailleurs », un agent de la Fonction publique d'État ou de la Fonction publique hospitalière n'a pas plus de chance de suivre une formation qu'un agent de la Fonction publique territoriale pris comme référence. En revanche, travailler dans le secteur privé diminue significativement la probabilité d'un départ en formation au regard de l'appartenance à la Fonction publique territoriale et aux autres fonctions publiques (- 6 points $c f$. modèle 1 , tableau 2$)$.

\section{$<$ INS TAB 02>}


Une autre hypothèse peut alors être avancée : la participation est peut-être plus forte dans la fonction publique car moins inégalitaire que dans le secteur privé.

\subsection{De moindres inégalités d'accès à la formation ?}

Le suivi d'une formation est très inégalitaire dans le secteur privé ; de nombreux travaux ont montré que cette sélectivité s'exerce au confluent des caractéristiques de l'emploi occupé par le salarié et de celles de l'entreprise qui l'emploie (Crocquey, 1995 ; Aventur, Hanchane, 1999 ; Michaudon, 2000).

Dans la fonction publique, la participation à la formation est relativement plus élevée mais recouvre des disparités de même nature que dans le privé : on se forme d'autant plus que l'on est diplômé, titulaire de son emploi, cadre et âgé de moins de 50 ans (Perez, 2002). Afin de consolider ce résultat, nous testons pour chacun des secteurs d'emploi, public et privé, l'influence de plusieurs variables d'intérêt sur la probabilité de suivre une formation. L'objectif consiste à comparer leurs poids relatifs dans chaque secteur pour déterminer si la probabilité de suivre une formation continue dépend des mêmes critères et s'exprime avec la même intensité dans les deux secteurs.

Ainsi, à caractéristique identique, un agent de la fonction publique a plus de chance de suivre une formation qu'un salarié du privé. Mais classiquement dans les deux secteurs, trois facteurs pèsent lourdement sur la probabilité de suivre une formation : le diplôme, la catégorie socioprofessionnelle et l'âge. ( $c f$. modèles 2 et 3 , tableau 2).

En revanche, la nature stable ou instable du contrat de travail ${ }^{12}$, l'ancienneté dans l'entreprise et le temps de travail n'ont que peu d'impact sur la probabilité d'accès à la formation dans la fonction publique, même s'il importe de noter qu'à l'instar du secteur privé, le fait de travailler à temps partiel pénalise la participation des femmes à la formation dans la fonction publique. Enfin, la chance de suivre une formation dans le public semble, là encore, ne pas dépendre de la fonction publique d'appartenance : les critères de sélectivité de la formation continue n'apparaissent pas significativement différents entre les fonctions publiques.

La probabilité de suivre une formation dépend donc essentiellement des mêmes variables dans la sphère publique et la sphère privé, même si les variables discriminantes sont moins nombreuses dans la première que dans la seconde. De même nature, ces inégalités sont-elles également de même ampleur ?

Dans un travail antérieur, on montrait que non seulement le différentiel de participation était toujours favorable à la fonction publique, à l'exception notable des contrats aidés, mais que les disparités intra catégorielles étaient moins grandes dans la fonction publique (Perez, 2002). Ceci est confirmé lorsqu'on raisonne «toutes choses égales par ailleurs ». La probabilité de suivre une formation pour un cadre du public est similaire à celle d'un cadre du privé. En revanche, un agent des professions intermédiaires du public a plus de « chance » de suivre une formation que son homologue du privé ; cet écart s'accroissant encore en faveur des employés et des ouvriers du public.

Nous observons bien l'effet du niveau de qualification sur la probabilité d'accès à la formation quel que soit le secteur, mais cet effet est renforcé dans le privé, au sens d'une aggravation des disparités. Autrement dit, à catégoric équivalente, pour être formé, il vaut mieux travailler dans le public que dans le privé ; et ce d'autant plus que l'on se situe en bas de la hiérarchie professionnelle.

\footnotetext{
${ }^{12}$ Nous laissons de côté les contrats aidés qui prévoient pour certains (tels le contrat de qualification) des périodes de formation. A cet égard, remarquons que les contrats aidés du secteur privé s'accompagnent bien plus fréquemment du suivi d'une formation que ceux de la fonction publique.
} 
Au total, la participation à la formation est globalement moins inégalitaire dans la fonction publique que dans le secteur privé, mais la sélectivité s'exerce selon les mêmes critères principaux que sont le diplôme, la catégorie socioprofessionnelle et l'âge. Un accès plus équitable à la formation ne peut expliquer seul la participation plus forte des agents de la fonction publique à la formation. Avant de conclure hâtivement à la plus grande prodigalité de la sphère publique, nous envisageons une autre piste explicative au travers de l'analyse des activités de travail et des contenus de formation.

En fait, moins que les caractéristiques de l'emploi occupé ou celles, individuelles, du formé, la spécificité des métiers et des fonctions exercés pourrait expliquer un recours plus fréquent à la formation dans la fonction publique. En outre, on émet l'hypothèse que les agents du public se forment autrement que ceux du privé, par exemple en privilégiant des formations à visée plus personnelle. Finalement, l'observation des contenus et des modalités de formation met en évidence plus de similarités que de différences entre les deux secteurs d'emplois.

\section{CONTENUS DES FORMATIONS : PLUS DE SIMILITUDES QUE DE DIFFÉRENCES}

\subsection{Des métiers et des fonctions requérant plus de formation ?}

Dans la fonction publique, les fonctions d'enseignement, de santé et d'information rassemblent la moitié des effectifs : $50 \%$ ont suivi une formation sur la période ( $c f$. tableau 3). Dans le secteur privé, la moitié des salariés se répartissent entre les fonctions «production, fabrication, chantiers » et " gestion, comptabilité »; les taux d'accès à la formation pour chacune d'elles s'élèvent respectivement à $23 \%$ et $40 \%$. La participation à la formation formelle est relativement faible pour les fonctions plus «manuelles » telles que la production, réparation, réglage et les services d'entretien et de surveillance, fonctions bien représentées dans le privé. Or, ces fonctions occupent moins du quart des agents de la fonction publique au profit des fonctions plus «intellectuelles » et qualifiées (recherche, santé...), associées à un accès plus large à la formation.

Toutefois, à fonction équivalente (et sous réserve que les qualifications mises en œuvre soient similaires), la participation à la formation est supérieure dans la fonction publique : par exemple, les salariés travaillant au guichet et à la saisie, -6 à $8 \%$ des effectifs salariés du privé et du public - n'ont pas eu un accès équitable à la formation, les seconds ayant plus fréquemment suivi une formation continue que les premiers ( $c f$. tableau 3 ).

\section{$<$ INS TAB 03>}

Ainsi, l'effet du secteur d'appartenance et celui de la fonction exercée semblent tous deux jouer en faveur de la sphère publique. Lorsque l'on raisonne «toutes choses égales par ailleurs », en contrôlant certaines caractéristiques de l'emploi occupé et du salarié, la contribution de la fonction exercée à la probabilité de suivre une formation est modeste, inférieure à celle du secteur d'appartenance. Seules les fonctions de «nettoyage, gardiennage » et « recherche, études » influent significativement sur cette probabilité, à la baisse pour la première fonction, et à la hausse pour la seconde (cf. modèle 1). La persistance de l'effet du secteur d'emploi, même après l'introduction de la fonction exercée, nous conduit à envisager à présent l'hypothèse de modalités et de contenus de formation différents selon le secteur d'appartenance.

\subsection{Des formations centrées sur les cœurs de métier}

Si les fonctionnaires se forment davantage que les salariés du privé, n'est-ce pas parce qu'ils se forment autrement et pour d'autres motifs ? En fait, à l'instar de ce qu'on observe dans le secteur privé, les formations sont essentiellement des stages financés par l'employeur visant l'adaptation à l'emploi. Les 
formations déclarées à but personnel (culturel, sportif...) ne représentent que $11 \%$ des formations, soit autant que dans le secteur privé ( $c f$. tableau 4).

\section{$<$ INS TAB 4}

Ainsi, dans la fonction publique, près de huit formations sur dix sont des stages (pour une présentation des types de formation, voir introduction au dossier). Majoritaire également dans le secteur privé, la part des stages y est toutefois inférieure de dix points. Plus précisément, la part des stages semble croitre avec la qualification des emplois : alors que trois formations sur quatre sont des stages dans la Fonction publique territoriale, $80 \%$ des formations se déroulent ainsi dans la Fonction publique d'État. Globalement, un tiers des stages sont consacrés à des formations pédagogiques d'éducateurs et de formateurs ainsi qu'à des formations médico-sociales. Les formations en situation de travail représentent un quart des formations suivies dans la Fonction publique territoriale, soit une part similaire à celle observée dans le secteur privé. La spécialité de formation la plus fréquemment associée est la bureautique et l'informatique. Enfin, dans les deux secteurs, l'autoformation ne représente qu'une part modeste des formations (4 à $5 \%$ des formations).

La diversité des métiers et des fonctions exercés entre les secteurs d'emploi, mais également à l'intérieur de chacun d'eux, se traduit par une pluralité des spécialités de formation. Mais les contenus de formation des agents de la fonction publique et des salariés du privé convergent sur plusieurs aspects. Tout d'abord, dans les deux secteurs, l'informatique est prépondérante en termes de spécialité de formation : $15 \%$ des formations concernant essentiellement la bureautique et l'utilisation de logiciels ont été suivies entre janvier 1999 et février 2000 par les agents de la fonction publique, soit plus de 550000 actions de formation. Viennent ensuite les formations centrées sur les " cœurs de métier » des secteurs. Ainsi, les formations pédagogiques de formateurs et d'éducateurs et les formations médico-sociales représentent chacune un peu plus de $13 \%$ des formations dans la fonction publique. Enfin, les formations relevant des ressources humaines et de l'encadrement qui ont été, comme l'informatique, encouragées par le ministère de la Fonction publique dans le cadre de la mise en œuvre de la «gestion prévisionnelle des emplois et des compétences », représentent $12 \%$ des formations.

Au total, et comme les salariés du privé, les agents de la fonction publique se forment essentiellement pour des motifs professionnels et sous des modalités assez proches de celles du secteur privé. Ce constat est confirmé par un travail plus approfondi qui croise différentes dimensions des formations et des caractéristiques des formés (Lowezanin, Perez, 2002). Ce travail permet de repérer plusieurs groupes de formation dans la fonction publique. Un premier ensemble a trait aux formations centrées sur les " cours de métier » visant l'adaptation à l'emploi de fonctionnaires titulaires. Ce groupe comprend les agents de catégorie A et B d'une part, exerçant des fonctions d'enseignement, recherche, gestion et comptabilité, et d'autre part, les agents de catégorie C, travaillant plutôt dans la Fonction publique territoriale et dont les formations relèvent des techniques industrielles, de l'hygiène et sécurité, des apprentissages de base. Un autre ensemble est caractérisé par des formations longues, générales et disciplinaires, associées à l'objectif d'un changement d'emploi ou l'obtention d'un diplôme. Il concerne des agents plutôt jeunes, non titulaires de leur emploi, finançant en partie eux-mêmes leur formation ${ }^{13}$.

Nous formulons enfin une dernière hypothèse, celle de l'influence de l'environnement professionnel sur les comportements à l'égard de la formation. Il est malaisé d'objectiver le rapport qu'un salarié entretient avec son milieu professionnel et la façon dont ce dernier peut agir sur le suivi d'une formation. C'est

Ceci fait écho à une étude en cours sur le recours et le rôle de la formation en début de carrière dans la fonction publique. Elle s'inscrit dans un projet de recherche commandité par le Ministère de la recherche et intitulé : "les déterminants des entrées dans les fonctions publiques: parcours du combattant ou pis aller ?" L'équipe de recherche comprend outre l'auteur, M. Lambert (Céreq), F. Audier (Matisse, Paris 1), D. Meurs (Ermes, Paris 2), V. Di Paola (Lest, Aix-Marseille 2) et S. Moullet (Céreq). 
pourquoi, à titre exploratoire, deux registres de variables vont être mobilisés : le premier registre a trait au caractère plus ou moins volontaire de l'entrée en formation, tandis que le second porte sur la connaissance des droits liés à la formation.

\section{DES RAPPORTS CONTRASTÉS À LA FORMATION}

\subsection{Des formations davantage à l'initiative du salarié dans le public ?}

L'origine de l'initiative de la formation, déclarée par le formé, donne une indication sur la façon dont il se représente, a posteriori, son rôle dans la procédure d'accès à la formation. Sous cette réserve, $36 \%$ des agents de la fonction publique, contre $19 \%$ des salariés du privé, déclarent être entièrement à l'initiative de leur formation à visée professionnelle ( $c f$. tableau 4 ). Dans ce domaine, il n'existe pas de différence significative entre les différentes fonctions publiques. Par ailleurs, les agents de la fonction publique déclarent moins souvent que leurs homologues du privé s'être vus imposer les formations suivies (respectivement $21 \%$ et $34 \%$ des formés). Cet écart ne semble pas lié au niveau de qualification puisque les agents de la Fonction publique territoriale, dont les niveaux de qualification sont similaires à ceux des salariés du privé, ont répondu comme les agents des autres fonctions publiques.

Gardons-nous d'associer étroitement l'initiative dont feraient preuve les salariés du public et l'appétence pour la formation. En effet, s'engager en formation suppose de connaître ses droits en la matière et d'y attacher l'espoir de meilleures perspectives professionnelles ou personnelles. Si l'on considère à la suite l'A.-L. Aucouturier que, "autant que la formation initiale, le contexte professionnel et institutionnel apparaît déterminant dans la création et la réalisation d'un projet de formation et dans la constitution même d'un "appétit" pour la formation », la sphère publique apparait alors comme un environnement plus propice que le secteur privé. En effet, les salariés du public se déclarent mieux informés de leurs droits que les salariés du privé et attendent davantage de la formation.

\subsection{Des agents mieux informés de leurs droits}

Ainsi, les agents de la fonction publique sont plus nombreux à connaître l'existence d'un plan de formation et, dans ce cas, à l'avoir effectivement consulté (61\% des agents informés de la fonction publique contre $25 \%$ de leurs homologues du privé). En outre, ils pensent, plus souvent que leurs homologues du secteur privé, avoir droit à un congé individuel de formation ou à un congé pour bilan de compétence. Ils sont aussi proportionnellement plus nombreux à considérer que l'employeur est tenu de former ses salariés pour les adapter à l'évolution de leur emploi.

Par ailleurs, les attentes à l'égard de la formation paraissent plus fortes dans le public que dans le privé. Globalement, plus de la moitié des agents de la fonction publique estiment que leurs besoins de formation seront assez voire très importants dans le futur, que ce soit pour leur vie professionnelle ou personnelle. Les salariés du privé semblent accorder un rôle plus modeste à la formation en exprimant des besoins de formation moindres.

L'apparente similarité des modalités de formation pourrait ainsi masquer des rapports à la formation plus contrastés selon les secteurs d'emploi. On rejoint ici les réflexions conduites par L. Rouban sur les attentes et les représentations des fonctionnaires face à la vie professionnelle. Comme les salariés du privé, les fonctionnaires se définissent en premier lieu par leur métier, avant leur diplôme ou leur organisation. Si les métiers évoluent au sein de la fonction publique, ils ne réduisent pas pour autant la distance socio-politique historiquement construite entre les deux secteurs. Du point de vue des attitudes, des divergences sensibles demeurent: «d'une manière générale, la participation sociopolitique des fonctionnaires est plus importante que celle des salariés du secteur privé [...] et les agents du secteur public sont, dans l'ensemble, davantage intéressés que les salariés du secteur privé par la politique et 
les questions économiques » (Rouban, 2001b). Les agents de la fonction publique, mieux informés de leurs droits, les exercent peut-être davantage.

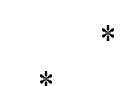

Dotés des mêmes dispositifs de formation, les agents de la fonction publique accèdent donc plus largement à la formation continue que leurs homologues du privé. Pour autant, ils ne se forment pas différemment. L'adaptation à l'emploi est, dans les deux secteurs, la principale attente de la formation et les contenus sont essentiellement centrés sur les activités professionnelles et l'adaptation aux transformations de l'organisation du travail. Les caractéristiques des agents, celles des emplois et des fonctions occupées, ne suffisent pas à expliquer la plus forte participation à la formation dans la fonction publique. L'existence de rapports contrastés à la formation peut constituer une piste explicative intéressante. La sphère publique apparaît, au travers de ce travail, comme un environnement plus propice au suivi d'une formation continue. Deux explications pourraient être avancées. La première renvoie à la formation continue comme «droit social », la sphère publique constituant un espace plus favorable à la connaissance et à l'exercice de ce droit. La seconde porte sur le rôle de la formation dans l'ensemble des marchés internes spécifiques qui constituent la fonction publique. La formation continue, notamment à travers la préparation aux concours, jouerait un rôle non négligeable dans l'organisation des mobilités, et l'espace des promotions se serait moins rétréci dans la fonction publique que dans le secteur privé. Si c'était le cas, ce serait de bon augure pour la gestion des tensions sur les recrutements et les mobilités. D'ailleurs, la formation est souvent présentée comme un des vecteurs essentiels de la modernisation de l'État, et comme le levier de la "gestion prévisionnelle des emplois et des compétences $\gg$ dans la fonction publique.

Cependant, l'énoncé de ces enjeux laisse dans l'ombre au moins une question cruciale. Comment concilier la «gestion prévisionnelle des emplois et des compétences » et le rôle joué par la formation en cours d'emploi, avec les principes qui régissent actuellement les mobilités dans la fonction publique ? Ainsi, dans le cadre des marchés internes, la formation en cours d'emploi est généralement spécifique et réside essentiellement dans l'expérience acquise au sein de l'entreprise. Combinée à l'ancienneté, l'expérience est reconnue par la possibilité d'une mobilité interne verticale. Dans le cadre spécifique de la fonction publique, l'acquisition de compétences en cours d'emploi par la formation est moins sélective que dans le privé. Elle est peut être même facilitée par le statut de la fonction publique qui assure à ceux qui en bénéficient la sécurité de l'emploi moyennant une nécessaire adaptation des connaissances et des qualifications au long de la carrière professionnelle. En revanche, la formation a peut-être moins de chance d'y constituer un appui pour une promotion interne. En effet, les carrières des agents demeurent étroitement liées aux règles de gestion des corps et à leur évolution démographique (Bieganski et alii, 1994), la primauté de l'ancienneté et du concours limitant les possibilités de valoriser les compétences acquises en formation. 


\section{Bibliographie}

Aucouturier A.-L. (2001), « La formation est arrivée près de chez vous... », Premières Informations et Premières Synthèses, Dares, ${ }^{\circ}$ 43.2, octobre.

Aventur F., Hanchane S. (1999), «Inégalités d'accès et pratiques de formation continue dans les entreprises françaises », Formation Emploi, ${ }^{\circ}$ 66, avril-juin, pp. 5-20.

Bentabet E., Gauthier C., Marion I. (2001), La formation professionnelle continue financée par les entreprises. Exploitation des déclarations fiscales des employeurs n²4-83. Année 1999. Céreq, Collection documents, $\mathrm{n}^{\circ} 160$, série Observatoire.

Béret P. (2002), «Formation professionnelle continue et carrières sexuées sur un marché interne », dans Baslé M. et alii. (éds.), Formation tout au long de la vie et carrières en Europe, Céreq, Série Séminaires, $\mathrm{n}^{\circ}$ 164, mai, pp. 99-111.

Bieganski R., Dubar C., Kalck P., Kleinkolt S. (1994), Les carrières dans la Fonction publique, rapport de recherche pour le ministère de la Fonction publique, Céreq, Études $n^{\circ} 66$, avril.

Commissariat général du Plan (1994), Gérer l'emploi public, Rapport du groupe technique «La politique de l'emploi dans les services publics » présidé par J. Rigaudiat, Février, 237 p.

Crocquey E. (1995), «La formation professionnelle continue : des inégalités d'accès et des effets sur la carrière peu importants à court terme », Travail et Emploi, $\mathrm{n}^{\circ}$ 65, pp. 61-68.

DGAFP (2001), La formation des agents de l'État en 1999. Enquête statistique sur les actions de formation réalisées par les ministères en faveur des agents de l'État, Volume 1 et 2, Bureau des statistiques, des études et de l'évaluation, avril.

Desbarat I. (2001), L'emploi précaire dans la fonction publique. Approche juridique. Communication aux VIIIèmes journées de sociologie du travail, LEST, Aix-en-Provence,21-23 juin.

Gautié J. (2002), Déstabilisation des marchés internes et gestion des âges sur le marché du travail : quelques pistes, Centre d'Etudes de l'Emploi, Document de travail, ${ }^{\circ} 15$, mars, $34 \mathrm{p}$.

Germe J.-F. (2001), «Au-delà des marchés internes : quelles mobilités, quelles trajectoires? », Formation Emploi, $\mathrm{n}^{\circ}$ 76, octobre-décembre, pp. 129-145.

Goux D., Maurin E. (1993), «La sécurité de l'emploi, une priorité croissante pour les diplômés », Économie et Statistiques, ${ }^{\circ}$ 251, pp. 67-77.

Goux D., Zamora P. (2001), « La formation en entreprise continue de se développer », Insee Premières, $\mathrm{n}^{\circ} 759$, février.

Kopel S. (2001), « Le déroulement de carrière des "surdiplômés" de la fonction publique : propositions pour une gestion différenciée », Politiques et management public, vol. 19, n 2, juin, pp. 25-43.

Lenoir H. (1996), « Fonction publique et formation : approche comparative », Actualité de la formation permanente, $\mathrm{n}^{\circ} 140$, pp. 15-22. 
Lenoir H. (1997), «La formation : quel outil de modernisation pour quelle Fonction publique ? », Education permanente, $\mathrm{n}^{\circ} 130$, pp. 131-141.

Lowezanin C., Perez C. (2002), La formation des agents de la Fonction publique. Exploitation de l'enquête Formation Continue 2000, rapport pour l'Instance d'évaluation de la politique de formation continue des agents de l'État, Commissariat général du Plan, janvier, 87p.

Michaudon H. (2000), «Investir en formation continue », Insee Premières, $n^{\circ}$ 697, février.

Ministère de la Fonction publique et de la réforme de l'État (2001), L'Observatoire de l'emploi public, Rapport anmuel, La Documentation française, juin, 111p.

Podevin G. (1999), "Formation-promotion sociale et professionnelle :un lien démocratique rompu ?» in Dubar C. et Gadéa C. (éds.), La promotion sociale en France, Presses Universitaires du Septentrion, pp. 213-226.

Perez C. (2002), La formation continue des agents de la fonction publique, Céreq-Bref, n¹84, mars.

Rouban L. (1995), La Fonction publique, La Découverte, 123 p.

Rouban L. (2001a), "Les fonctionnaires face à la vie professionnelle : attentes et représentations », La Revie administrative, $\mathrm{n}^{\circ} 322$, juillet-août, pp. 417-424.

Rouban L. (2001b), Les fonctionnaires, Le Cavalier Bleu Éditions, Coll. « Idées reçues », 127 p.

Singly F. (de), Thélot C. (1988), Gens du privé, gens du public, Dunod, 256 p. 


\section{Les trois fonctions publiques et leurs salariés}

La fonction publique d'État, la fonction publique territoriale et la fonction publique hospitalière sont des fonctions publiques de carrière ; elles offrent la possibilité à leurs agents de progresser tout au long de la vie professionnelle. Elles sont organisées en corps (Fonction publique d'État et Fonction publique hospitalière) ou en cadres d'emplois (Fonction publique territoriale) rassemblant les fonctionnaires soumis au même statut (par exemple les inspecteurs des impôts, les attachés d'administration...). En outre, le corps (ou le cadre d'emploi) est séparé de l'emploi : les agents sont susceptibles d'occuper tous les emplois de leur corps d'appartenance auquel leur grade leur donne vocation. Chaque corps (ou cadre d'emploi) comprend des classes ou des grades divisés en échelons. Ils situent les agents dans la hiérarchie administrative et balisent leur progression salariale.

Le recrutement par concours constitue le droit commun pour accéder à la fonction publique. Les concours externes sont ouverts aux candidats justifiant des diplômes requis pour la catégorie correspondante. Ainsi, un diplôme de niveau licence est requis pour postuler à un concours externe de la catégorie $\mathrm{A}$, le baccalauréat pour la catégorie $\mathrm{B}$ et le brevet pour la catégorie $\mathrm{C}$. Les concours internes interviennent en cours de carrière et concernent des agents présentant l'ancienneté requise pour changer de grade ou de corps. Enfin, depuis la loi du 3 janvier 2001 relative à la résorption de l'emploi précaire et à la modernisation des recrutements dans la fonction publique, les «troisièmes concours »sont ouverts aux personnes justifiant d'une activité professionnelle privée ou associative $(*)$.

Les agents de la « nébuleuse publique », pour reprendre une expression de F. de Singly et C. Thélot, ont été repérés à partir de l'enquête Emploi en privilégiant le caractère privé ou public de l'établissement. L'activité économique de l'emploi occupé et la profession exercée ont été utilisées en complément afin de distinguer les trois fonctions publiques. Au total, notre effectif pondéré d'agents de la fonction publique (qu'ils soient fonctionnaires, contractuels ou agents publics) est estimé à 5260000 qui se répartissent comme suit : 2873000 dans la Fonction publique d'État (y compris les enseignants de l'enseignement privé sous contrat), 1553000 dans la Fonction publique territoriale et près de 833000 dans la Fonction publique hospitalière.

$(*)$ : Pour en savoir plus : Ministère de la Fonction publique et de la réforme de l'État, rapport annuel de l'observatoire de l'emploi public, juin 2001 . 


\section{Graphique 1}

Répartition des agents de la fonction publique et des salariés du privé par diplôme*

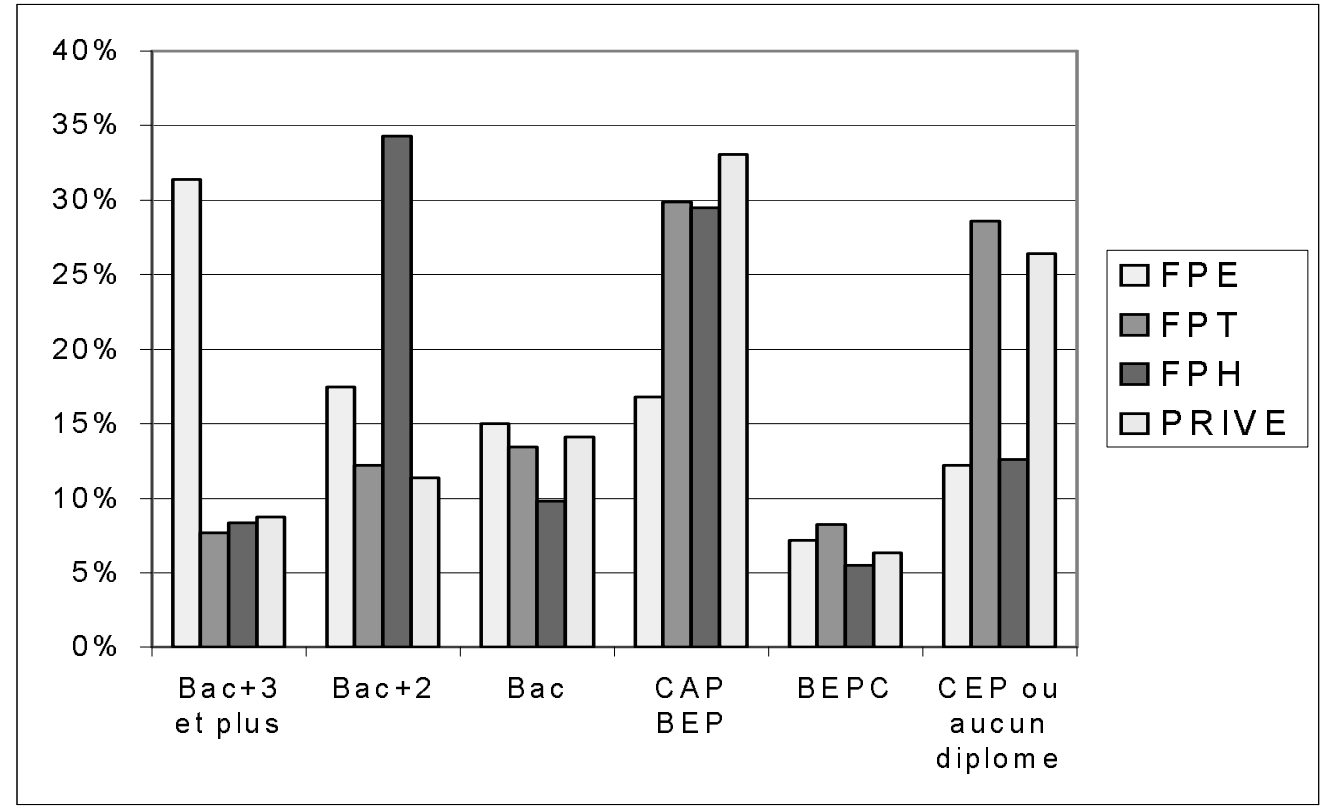

Source : FC2000. Traitement : Céreq.

Champ : les salariés du public et du privé.

*Il s'agit du plus haut diplôme obtenu par l'enquêté. 


\section{Graphique 2}

Répartition des durées cumulées de formation sur les 14 derniers mois dans la fonction publique et le secteur privé

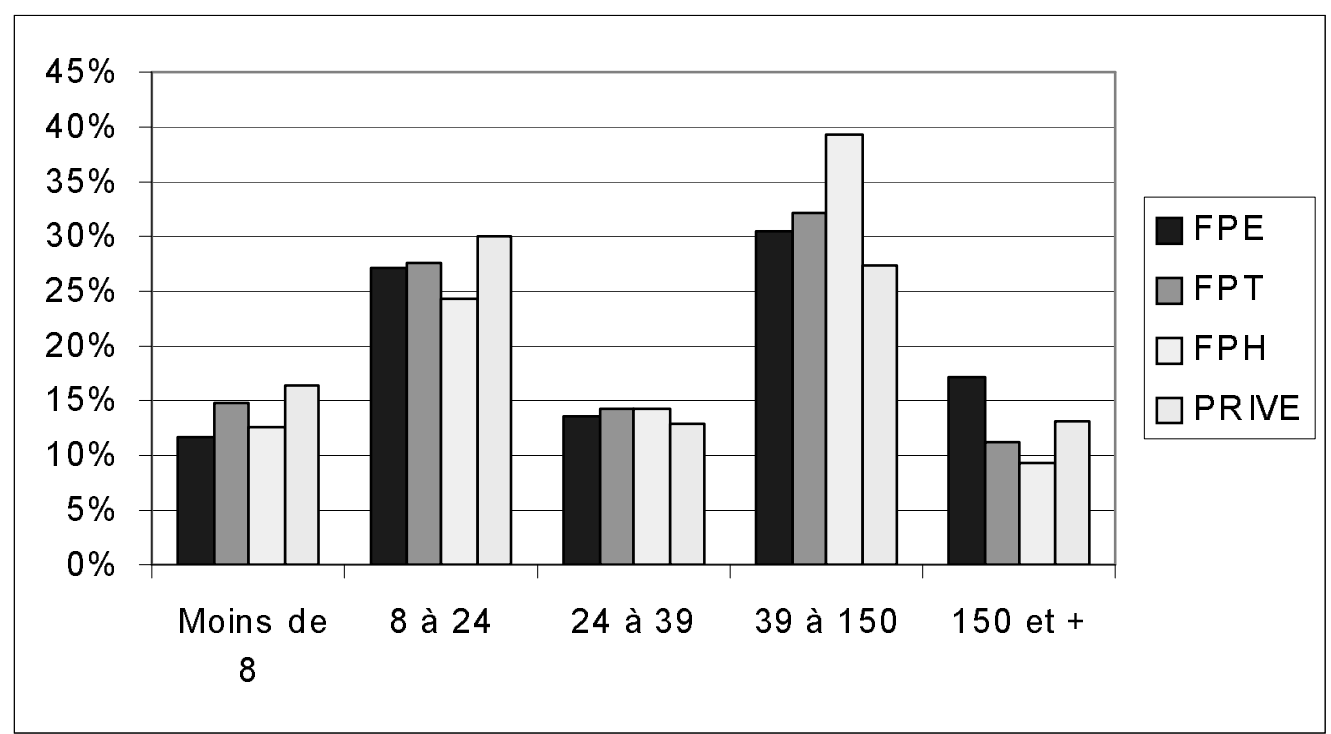

Source : FC 2000. Traitement : Céreq

Champ : les salariés du public et du privé ayant suivi au moins une formation entre janvier 1999 et février 2000 . 
Tableau 1

Les durées cumulées de formation sur la période janvier 1999-février 2000

\begin{tabular}{|l|l|l|}
\hline & Fonction publique & Secteur privé \\
\hline $75 \%$ des salariés ont connu moins de... & 78 heures & 64 heures \\
\hline $50 \%$ des salariés ont connu moins de... & 39 heures & 32 heures \\
\hline $25 \%$ des salariés ont connu moins de... & 16 heures & 16 heures \\
\hline
\end{tabular}

Exemple de lecture : un quart des agents de la fonction publique et un quart des salariés du privé ont suivi moins de 16 heures de formation entre janvier 1999 et février 2000.

Source : enquête « Formation continue $2000 »$. Traitement : Céreq

$<\mathrm{NP}>$ 
Tableau 2

Logit binomial sur les déterminants de l'accès à la formation des salariés du public et du privé*

\begin{tabular}{|c|c|c|c|}
\hline & Modèle 1 & Modèle 2 & Modèle 3 \\
\hline Champ & Tous les salariés & $\begin{array}{c}\text { Agents de la } \\
\text { fonction publique }\end{array}$ & $\begin{array}{c}\text { Salariés du secteur } \\
\text { privé }\end{array}$ \\
\hline Nombre d'observations & 16952 & 4713 & 12239 \\
\hline $\begin{array}{l}\text { Probabilité de la situation de } \\
\text { référence }\end{array}$ & $22 \%$ & $25 \%$ & $20 \%$ \\
\hline $\begin{array}{l}\text { Age } \\
15-24 \text { ans } \\
25-34 \text { ans } \\
35-44 \text { ans } \\
45-54 \text { ans } \\
55 \text { ans et plus } \\
\end{array}$ & $\begin{array}{c}+5 \\
- \\
r e ́ f . \\
-3 \\
-12 \\
\end{array}$ & $\begin{array}{c}- \\
- \\
\text { réf. } \\
-3^{*} \\
-13 \\
\end{array}$ & $\begin{array}{l}+6 \\
+2 * \\
\text { réf. } \\
-3 \\
-10 \\
\end{array}$ \\
\hline $\begin{array}{l}\text { Diplôme } \\
\text { Bac }+3 \text { et plus } \\
\text { Bac }+2 \\
\text { Bac } \\
\text { Cap-Bep } \\
\text { BEPC } \\
\text { Aucun diplôme } \\
\end{array}$ & $\begin{array}{l}+20 \\
+19 \\
+14 \\
+8 \\
+8 \\
\text { réf. }\end{array}$ & $\begin{array}{l}+29 \\
+28 \\
+21 \\
+14 \\
+16 \\
\text { réf. }\end{array}$ & $\begin{array}{l}+21 \\
+20 \\
+15 \\
+8 \\
+7 \\
\text { réf. }\end{array}$ \\
\hline $\begin{array}{l}\text { Sexe*temps de travail } \\
\text { Homme*temps complet } \\
\text { Homme*temps partiel } \\
\text { Femme*temps complet } \\
\text { Femme*temps partiel }\end{array}$ & $\begin{array}{l}\text { réf. } \\
-6 \\
-1 * \\
-5\end{array}$ & $\begin{array}{l}\text { réf. } \\
- \\
- \\
-4^{*}\end{array}$ & $\begin{array}{l}r e ́ f . ~ \\
-7 \\
-2 * \\
-6\end{array}$ \\
\hline $\begin{array}{l}\text { Nature du contrat } \\
\text { CDI, titulaire } \\
\text { CDD, intérim, non titulaire } \\
\text { Contrats aidés }\end{array}$ & $\begin{array}{c}\text { réf. } \\
- \\
+19 \\
\end{array}$ & $\begin{array}{c}\text { réf. } \\
- \\
+7^{*} \\
\end{array}$ & $\begin{array}{l}\text { réf. } \\
-2^{*} \\
+30 \\
\end{array}$ \\
\hline $\begin{array}{l}\text { PCS } \\
\text { Cadres } \\
\text { Professions intermédiaires } \\
\text { Employés } \\
\text { Ouvriers } \\
\end{array}$ & $\begin{array}{l}+16 \\
+15 \\
+9 \\
\text { réf. }\end{array}$ & $\begin{array}{c}+11 \\
+12 \\
+7 \\
\text { réf. }\end{array}$ & $\begin{array}{c}+20 \\
+17 \\
+9 \\
\text { réf. }\end{array}$ \\
\hline $\begin{array}{l}\text { Ancienneté dans l'entreprise } \\
<1 \text { an } \\
\text { de } 1 \text { à } 3 \text { ans } \\
\text { de } 3 \text { à } 5 \text { ans } \\
\text { de } 5 \text { à } 10 \text { ans } \\
\text { de } 10 \text { à } 20 \text { ans } \\
20 \text { ans et plus }\end{array}$ & $\begin{array}{c}- \\
-3 \\
\text { réf. } \\
- \\
+3^{*} \\
+5\end{array}$ & $\begin{array}{c}-5^{*} \\
- \\
\text { réf. } \\
- \\
- \\
- \\
\end{array}$ & $\begin{array}{l}- \\
-3 * \\
\text { réf. } \\
- \\
+4 \\
+5\end{array}$ \\
\hline $\begin{array}{l}\text { Secteur d'emploi } \\
\text { Fonction publique d'État } \\
\text { Fonction publique territoriale } \\
\text { Fonction publique hospitalière } \\
\text { Secteur privé } \\
\end{array}$ & $\begin{array}{c}- \\
\text { réf. } \\
- \\
-6\end{array}$ & $\begin{array}{c}- \\
\text { réf. } \\
-\end{array}$ & \\
\hline $\begin{array}{l}\text { Fonction exercée } \\
\text { Enseignement, santé } \\
\text { Production, fabrication, .. } \\
\text { Installation, entretien } \\
\text { Nettoyage, gardiennage } \\
\text { Manutention, magasinage } \\
\text { Guichet, saisie } \\
\text { Gestion, comptabilité } \\
\text { Recherche, études } \\
\text { Direction générale } \\
\end{array}$ & $\begin{array}{c}- \\
- \\
- \\
-10 \\
\text { réf. } \\
\\
+8 \\
- \\
\end{array}$ & & \\
\hline $\begin{array}{l}\text { Taille d'entreprise } \\
\text { Moins de } 10 \text { sal. }\end{array}$ & & & -9 \\
\hline
\end{tabular}




\begin{tabular}{|l||c||c|}
\hline De 10 à 49 sal. & & -8 \\
De 50 à 499 sal. & & -2 \\
Plus de 500 sal. & & réf. \\
\hline
\end{tabular}

Source : Enquête FC 2000. Traitement : Céreq

Résultats significatifs à $1 \%$; * à $5 \%$; - non significatif à $5 \%$.

* a réalisé au moins une formation au cours des 14 derniers mois.

Lecture : pour chacun des modèles, ce tableau indique les différences de probabilité d'une modalité considérée par rapport à la probabilité globale du modèle.

Dans le modèle 1, la situation de référence est celle d'un homme travaillant à temps complet, ouvrier en CDI (ou titulaire), dans la Fonction publique territoriale, âgé de 35 à 44 ans, sans diplôme, ayant de 3 à 5 ans d'ancienneté dans son entreprise et exerçant des fonctions de manutention, magasinage. Sa probabilité d'avoir suivi une formation dans les quatorze derniers mois est de $22 \%$. Le fait de travailler dans le secteur privé, toutes choses égales par ailleurs, fait chuter cette probabilité de 6 points. Ce sont donc les écarts (16-22=-6) qui sont présentés dans ce tableau.

Le modèle 2 ne concerne que les agents de la fonction publique et n'intègre pas d'informations sur la fonction exercée et la taille de l'entreprise. A ceci près, la situation de référence est identique à la précédente.

Le modèle 3 concerne les salariés du secteur privé. La probabilité d'accès à la formation de l'individu de référence est de $20 \%$. À caractéristique identique, le fait de travailler dans une entreprise de moins de 10 salariés fait chuter la probabilité d'accès à la formation de 9 points.

$<\mathrm{NP}>$ 
Tableau 3

Fonctions exercées et accès à la formation des salariés du public et du privé

\begin{tabular}{|c|c|c|c|c|}
\hline & \multicolumn{2}{|c|}{ Fonction publique } & \multicolumn{2}{|c|}{ Secteur privé } \\
\hline Fonctions & $\begin{array}{l}\text { Taux } \\
\text { d'accès à } \\
\text { la } \\
\text { formation( } \\
\% \text { ) }\end{array}$ & $\begin{array}{l}\text { Part dans } \\
\text { la } \\
\text { population } \\
(\%)\end{array}$ & $\begin{array}{l}\text { Taux } \\
\text { d'accès } \\
(\%)\end{array}$ & $\begin{array}{l}\text { Part dans } \\
\text { la } \\
\text { population } \\
(\%)\end{array}$ \\
\hline $\begin{array}{l}\text { Enseignement, } \\
\text { santé }\end{array}$ & 50 & 50 & 38 & 11 \\
\hline $\begin{array}{l}\text { Production, } \\
\text { fabrication, .. }\end{array}$ & 40 & 2 & 23 & 26 \\
\hline $\begin{array}{l}\text { Installation, } \\
\text { entretien }\end{array}$ & 33 & 7 & 32 & 7 \\
\hline $\begin{array}{l}\text { Nettoyage, } \\
\text { gardiennage }\end{array}$ & 20 & 10 & 13 & 8 \\
\hline $\begin{array}{l}\text { Manutention, } \\
\text { magasinage }\end{array}$ & 24 & 2 & 25 & 9 \\
\hline Guichet, saisie & 46 & 8 & 35 & 6 \\
\hline $\begin{array}{l}\text { Gestion, } \\
\text { comptabilité }\end{array}$ & 54 & 15 & 40 & 26 \\
\hline $\begin{array}{l}\text { Recherche, } \\
\text { études }\end{array}$ & 61 & 4 & 57 & 6 \\
\hline $\begin{array}{l}\text { Direction } \\
\text { générale }\end{array}$ & 61 & 2 & 49 & 1 \\
\hline Ensemble & 47 & 100 & 32 & 100 \\
\hline
\end{tabular}

Exemple de lecture : $11 \%$ des salariés du privé exercent une fonction «enseignement, santé... ». $38 \%$ d'entre eux ont suivi une formation entre janvier 1999 et février 2000.

Source : enquête « Formation continue $2000 »$. Traitement : Céreq.

Les taux d'accès présentent les proportions de personnes ayant suivi au moins une action de formation, d'au moins trois heures, quel que soit le but (explicitement professionnel ou plus personnel) et les modalités (stages, autoformation, formation en situation de travail, alternance), entre janvier 1999 et février 2000 .

$<\mathrm{NP}>$ 
Tableau 4

Répartition des formés du public et du privé selon les caractéristiques de leur formation

\begin{tabular}{|c|c|c|}
\hline & $\begin{array}{l}\text { Fonction } \\
\text { publique }\end{array}$ & Secteur privé \\
\hline \multicolumn{3}{|l|}{ Type } \\
\hline Alternance & 1 & 3 \\
\hline Stages & 78 & 68 \\
\hline $\begin{array}{l}\text { FEST(formation } \\
\text { en situation de } \\
\text { travail) }\end{array}$ & 16 & 25 \\
\hline Autoformation & 5 & 4 \\
\hline \multicolumn{3}{|l|}{ Attente } \\
\hline $\begin{array}{l}\text { Adaptation à } \\
\text { l'emploi }\end{array}$ & 74 & 76 \\
\hline $\begin{array}{l}\text { Obtention ou } \\
\text { changement } \\
\text { d'emploi }\end{array}$ & 7 & 7 \\
\hline $\begin{array}{l}\text { Diplôme ou } \\
\text { qualification }\end{array}$ & 8 & 7 \\
\hline $\begin{array}{l}\text { But culturel, } \\
\text { sportif, } \\
\text { associatif... }\end{array}$ & 11 & 10 \\
\hline \multicolumn{3}{|l|}{$\begin{array}{l}\text { Financeur } \\
\text { principal** }\end{array}$} \\
\hline Employeur & 73 & 83 \\
\hline Individu & 6 & 4 \\
\hline Etat, ANPE. & 13 & 5 \\
\hline $\begin{array}{l}\text { Autres } \\
\text { (fournisseur, } \\
\text { association...) }\end{array}$ & 8 & 8 \\
\hline \multicolumn{3}{|l|}{ Initiative* } \\
\hline Employeur seul & 33 & 49 \\
\hline Individu seul & 36 & 19 \\
\hline $\begin{array}{l}\text { Employeur et } \\
\text { individu }\end{array}$ & 27 & 24 \\
\hline $\begin{array}{l}\text { Autres (services } \\
\text { pour l'emploi...) }\end{array}$ & 4 & 8 \\
\hline
\end{tabular}

*Des formations à visée professionnelle

Exemple de lecture : $78 \%$ des formés de la fonction publique ont suivi un stage contre $68 \%$ de leurs homologues du privé.

Champ : les salariés du public et du privé ayant suivi au moins une formation entre janvier 1999 et février 2000.

Source : Enquête FC 2000. Traitement : Céreq

$<\mathrm{NP}>$ 